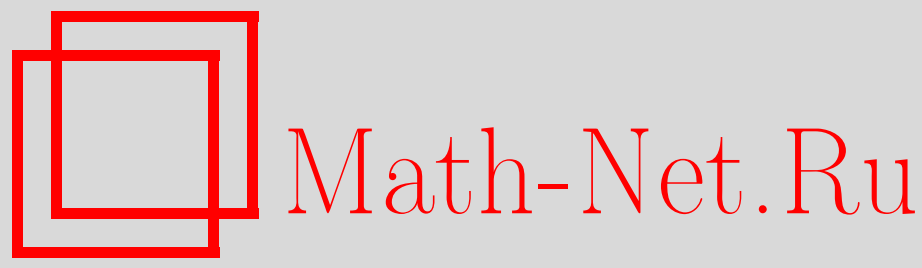

A. А. Часовских, Проблема полноты для класса линейно-автоматных функций, Дискрет. матем., 2015, том 27, выпуск 2, 134-151

DOI: https://doi.org/10.4213/dm1330

Использование Общероссийского математического портала Math-Net.Ru подразумевает, что вы прочитали и согласны с пользовательским соглашением http: //www . mathnet.ru/rus/agreement

Параметры загрузки:

IP: 3.85 .183 .62

26 апреля 2023 г., 15:25:04

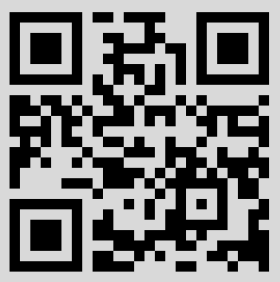




\title{
Проблема полноты для класса линейно-автоматных функций
}

\author{
() 2015 г. А. А. Часовских*
}

\begin{abstract}
Рассматриваются классы линейно-автоматных функций над конечными полями с операциями композиции (суперпозиции и обратной связи). Для этих классов получен алгоритм проверки полноты конечных систем. Таким образом, обобщается результат, известный для классов линейно-автоматных функций над простыми конечными полями.
\end{abstract}

Ключевые слова: конечный автомат, линейно-автоматная функция, операции композиции, операции суперпозиции, обратная связь, проблема полноты, критерий полноты, сумматор, задержка.

\section{1. Постановка задачи. Необходимое условие К-полноты}

Зафиксируем простое число $p$ и натуральное число $m$. Через $k$ обозначим число $p^{m}$, а через $E_{k}[\xi]$ обозначим множество всех многочленов от переменной $\xi$ над полем $E_{k}$. Для множества всех многочленов из $E_{k}[\xi]$ с ненулевым свободным членом будем использовать обозначение $E_{k}^{\prime}[\xi]$. Поле отношений многочленов из $E_{k}[\xi]$ обозначаем $E_{k}(\xi)$, а его подкольцо

$$
\left\{\frac{u(\xi)}{v(\xi)} \mid u(\xi) \in E_{k}[\xi], v(\xi) \in E_{k}^{\prime}[\xi]\right\}
$$

будем обозначать $E_{k}^{\prime}(\xi)$. Множество всех формальных степенных рядов по переменной $\xi$ с коэффициентами из $E_{k}$ будем обозначать $R_{k}(\xi)$.

Пусть $n \in \mathbb{N}$. Отображение $f\left(x_{1}, x_{2}, \ldots, x_{n}\right)$ из $R_{k}^{n}(\xi)$ в $R_{k}(\xi)$ называется линейно-автоматной функцией (л.-а. функцией) над $E_{k}$, если найдутся такие $\mu_{i}, \quad \mu_{i} \in E_{k}^{\prime}(\xi), i=0,1, \ldots, n$, что для любых $\alpha_{i}, \quad \alpha_{i} \in R_{k}(\xi), i=1,2, \ldots, n$, выполнено равенство

$$
f\left(\alpha_{1}, \alpha_{2}, \ldots, \alpha_{n}\right)=\sum_{i=1}^{n} \mu_{i} \alpha_{i}+\mu_{0}
$$

где операции "+"и "."индуцированы операциями в $E_{k}$. Поэтому л.-а. функция $f\left(x_{1}, x_{2}, \ldots, x_{n}\right)$ задается выражением $\sum_{i=1}^{n} \mu_{i} x_{i}+\mu_{0}$.

Как показано в [2], л.-а. функция реализуется линейной последовательной машиной. При этом ряд $\alpha_{i}, \alpha_{i}=\sum_{i=0}^{\infty} \alpha_{i}(t) \xi^{t}$, соответствует последовательности $\alpha_{i}(0)$,

* Место работы: МГУ им. М. В. Ломоносова, e-mail: chasovskikh@mail.ru 
$\alpha_{i}(1), \ldots$, подаваемой на $i$-й вход машины, а параметр $t$ определяет момент времени подачи сигнала $\alpha_{i}(t)$. Множество всех л.-а. функций над $E_{k}$ обозначим $\mathfrak{L}_{k}$.

Приведем примеры л.-а. функций из $\mathfrak{L}_{k}$ (см. [2]).

(1) Сумматор от $n$ переменных: $x_{1}+x_{2}+\ldots+x_{n}, n \in \mathbb{N}$.

(2) Усилители: $a \cdot x, a \in E_{k} \backslash\{0\}$.

(3) Константа: $\mu, \mu \in E_{k}^{\prime}(\xi)$.

(4) Задержка с начальным состоянием $a, a \in E_{k}: \xi \cdot x+a$.

В классе $\mathfrak{L}_{k}$ рассмотрим операторы замыкания $S$ и $K$ по операциям суперпозиции и композиции соответственно [4; стр. 155].

Пусть $M \subseteq \mathfrak{L}_{k}$. Множество $M$ называется $K$-полным, если $K(M)=\mathfrak{L}_{k}$. Множество $M$ называется $K$-базисом, если оно $K$-полно и $K(M \backslash\{f\}) \neq \mathfrak{L}_{k}$ для любой $f \in M$.

Пусть

$$
B=\left\{\begin{array}{ccc}
\left\{x_{1}+x_{2}, \xi \cdot x, 1\right\}, & \text { если } & m=1 \\
\left\{x_{1}+x_{2}, a \cdot x, \xi \cdot x, 1\right\} \mid a- & & \\
\text { примитивный элемент [6] поля } E_{k}, & \text { если } & m>1 .
\end{array}\right.
$$

Нетрудно доказать следующее утверждение.

Лемма 1. Множество л.-а. функиий $B$ является $K$-базисом в $\mathfrak{L}_{k}$.

Цель настоящей работы - построить алгоритм, позволяющий по заданному конечному множеству л.-а. функций проверять, является ли оно $K$-полным.

Через $\mu(0)$ будем обозначать свободный член ряда $\mu(\xi), \mu(\xi) \in R_{k}(\xi)$.

Рассмотрим следующие множества л.-а. функций.

Пусть $s \in E_{k}$,

$$
\begin{aligned}
T_{s}= & \left\{f\left(x_{1}, x_{2}, \ldots, x_{n}\right) \mid n \in \mathbb{N}, f \in \mathfrak{L}_{k},\right. \text { из } \\
& f\left(x_{1}, x_{2}, \ldots, x_{n}\right)=\sum_{i=1}^{n} \mu_{i} x_{i}+\mu_{0} \text { следует } \\
& \left.\sum_{i=1}^{n} \mu_{i}(0) \cdot s+\mu_{0}(0)=s\right\} .
\end{aligned}
$$

Таким образом, $T_{s}$ - множество всех л.-а. функций, сохраняющих число $s$ в начальный момент времени.

Далее, положим

$$
\begin{aligned}
V_{1}= & \left\{f\left(x_{1}, x_{2}, \ldots, x_{n}\right) \mid n \in \mathbb{N}, f \in \mathfrak{L}_{k},\right. \\
& f\left(x_{1}, x_{2}, \ldots, x_{n}\right)=\sum_{i=1}^{n} \mu_{i} x_{i}+\mu_{0}, \\
& \text { среди чисел } \mu_{i}(0), i=1,2, \ldots, n, \\
& \text { не более одного отличного от нуля }\},
\end{aligned}
$$

т.е. $V_{1}$ - множество всех л.-а. функций, существенно зависящих в начальный момент не более чем от одной переменной.

Множество $M, M \subseteq \mathfrak{L}_{k}$, называется $K$-замкнутым классом, если выполнено равенство $M=K(M)$. 
Как нетрудно видеть, л.-а. функции, рассматриваемые в этой работе, в первый момент времени реализуют квазилинейные функции над полем $E_{k}$ [8], [9]. В работе [9] были определены все замкнутые подклассы класса $L_{k}$ всех квазилинейных функций, не содержащиеся в классе одноместных функций. Это множество классов квазилинейных функций обозначим $J_{k, 0}$.

Каждому классу $\Theta, \Theta \in J_{k, 0}$, сопоставим класс $\Theta^{\prime}$ всех л.-а. функций, каждая из которых в начальный момент времени реализует функцию из $\Theta$. Положим:

$$
J^{\prime}=\left\{\Theta^{\prime} \mid \Theta \in J_{k, 0}\right\} \cup V_{1} .
$$

Для каждого $s, s \in E_{k}$, выполнено: $T_{s} \in J^{\prime}$.

Используя результат работы [9], несложно доказать следующее утверждение.

Теорема 1. Каждый элемент множества $J_{k}^{\prime}$ является K-замкнутым классом, не совпадающим с $\mathfrak{L}_{k}$.

Пусть л.-а. функция $f\left(x_{1}, x_{2}, \ldots, x_{n}\right)$ определена равенством (1). Тогда положим:

$$
U(f)=\left\{\mu_{i} \mid i=1,2, \ldots, n\right\},
$$

а для множества л.-а. функций $M$

$$
U(M)=\cup_{f \in M} U(f) .
$$

Таким образом, $U(M) \subseteq E_{k}^{\prime}(\xi)$.

Теорема 2. Для множества $M, M \subseteq \mathfrak{L}_{k}$, равенство

$$
K(M)=\mathfrak{L}_{k}
$$

выполнено в точности тогда, когда имеют место следующие свойства:

для любого $\Theta \in J_{k}^{\prime}$

$$
\begin{gathered}
M \nsubseteq \Theta, \\
U(K(M))=E_{k}^{\prime}(\xi) .
\end{gathered}
$$

Для доказательства полноты множества $M$, удовлетворяющего условиям (3) и (4), нам понадобится вспомогательное утверждение.

Лемма 2. Если для некоторого $M, M \subseteq \mathfrak{L}_{k}$, выполнены условия (3) и (4), то:

$$
x_{1}+x_{2}+\ldots+x_{p+1} \in K(M),
$$

для любого $\mu, \mu \in E_{k}^{\prime}(\xi)$, в $K(M)$ содержится функиия

$$
x_{1}+\mu \cdot x_{2}+\mu \cdot x_{3}+\ldots+\mu \cdot x_{p+1},
$$

в $K(M)$ содержатся такие константы $\nu_{0} u \nu_{0}^{\prime}$, что

$$
\nu_{0}(0)=0, \quad \nu_{0}^{\prime}(0) \neq 0 .
$$


Доказательство. Рассмотрим множество $M, M \subseteq \mathfrak{L}_{k}$, обладающее свойствами (3) и (4).

Тогда найдется л.-а. функция $f\left(x_{1}, x_{2}, \ldots, x_{n}\right), f \in M \backslash V_{1}$. Для некоторых $\mu_{i}$, $\mu_{i} \in E_{k}^{\prime}(\xi), i=0,1, \ldots, n$, имеет место равенство (1). Не ограничивая общности рассуждений, будем предполагать, что $\mu_{i}(0) \neq 0, i=1,2$. Найдутся такие л.-а. функции $g_{i}, g_{i} \in K(M), i=1,2$, что $\mu_{i}^{-1} \in U\left(g_{i}\right)$. Из функции $g_{i}$, используя операции переименования и отождествления переменных, получим л.-а. функцию $g_{i}^{\prime}\left(x_{i}, x_{3}\right), g_{i}^{\prime}\left(x_{i}, x_{3}\right)=\mu_{i}^{-1} \cdot x_{i}+g_{i}^{\prime \prime}\left(x_{3}\right), i=1,2$. Тогда, обозначив л.-а. функцию $f\left(g_{1}^{\prime}\left(x_{1}, x_{3}\right), g_{2}^{\prime}\left(x_{2}, x_{3}\right), x_{3}, \ldots, x_{3}\right)$ через $h\left(x_{1}, x_{2}, x_{3}\right)$, для некоторых $\mu_{i}^{\prime}, i=0,1$, имеем: $h\left(x_{1}, x_{2}, x_{3}\right)=x_{1}+x_{2}+\mu_{1}^{\prime} \cdot x_{3}+\mu_{0}^{\prime}$. Имеет место равенство

$$
h\left(x_{1}, h\left(x_{2}, \ldots h\left(x_{p}, x_{p+1}, x\right), \ldots x\right), x\right)=x_{1}+x_{2}+\ldots+x_{p+1} .
$$

Поэтому включение (5) доказано.

Далее, для каждого $\mu, \mu \in E_{k}^{\prime}(\xi)$, в $K(M)$ содержится такая л.-а. функция $f_{\mu}\left(x, x_{1}\right)$, что для некоторых $\mu_{1}$ и $\mu_{0}$ из $E_{k}^{\prime}(\xi)$ выполнено

$$
f_{\mu}\left(x, x_{1}\right)=\mu \cdot x+\mu_{1} \cdot x_{1}+\mu_{0} .
$$

Имеет место равенство

$$
x_{1}+f_{\mu}\left(x_{2}, x\right)+f_{\mu}\left(x_{3}, x\right)+\ldots+f_{\mu}\left(x_{p+1}, x\right)=x_{1}+\mu \cdot x_{2}+\mu \cdot x_{3}+\ldots+\mu \cdot x_{p+1},
$$

доказывающее утверждение (6).

Из предположения (3) следует, что в $K(M)$ содержатся функции $\tilde{\eta}_{0}$ и $\tilde{\eta}_{0}^{\prime}$, реализующие в первый момент времени константы 0 и 1 , соответственно. Отождествляя переменные каждой из этих функций, затем применяя операцию обратной связи к полученным одноместным функциям, получаем константы $\eta_{0}$ и $\eta_{0}^{\prime}$, удовлетворяющие (7). Лемма доказана.

Доказательство теоремы. Пусть выполнено (2). Тогда свойство (3) следует из теоремы 1 , а справедливость (4) очевидна.

Пусть для множества $M, M \subseteq \mathfrak{L}_{k}$, выполнены условия (3) и (4). Согласно лемме 2 , в $K(M)$ содержатся такие константы $\nu_{0}$ и $\nu_{0}^{\prime}$, что $\nu_{0}(0)=0, \nu_{0}^{\prime}(0) \neq 0$. Тогда для некоторых многочленов $u(\xi), u^{\prime}(\xi), v(\xi), v^{\prime}(\xi)$ из $E_{k}[\xi]$ имеют место равенства

$$
\nu_{0}=\frac{\xi \cdot u(\xi)}{1+\xi \cdot v(\xi)}, \nu_{0}^{\prime}=\frac{-1+\xi \cdot u^{\prime}(\xi)}{v^{\prime}(\xi)} .
$$

Справедливо тождество

$$
\left(1-\xi \cdot u^{\prime}(\xi)\right)(1+\xi \cdot v(\xi)) \nu_{0}+\xi \cdot u(\xi) v^{\prime}(\xi) \nu_{0}^{\prime}=0
$$

Через $u_{1}(\xi)$ и $u_{2}(\xi)$ обозначим многочлены $\xi\left(v(\xi)-u^{\prime}(\xi)-\xi v(\xi) u^{\prime}(\xi)\right)$ и $\xi u(\xi) v^{\prime}(\xi)$, соответственно. Имеем тождество:

$$
\nu_{0}+u_{1}(\xi) \nu_{0}+u_{2}(\xi) \nu_{0}^{\prime}=0
$$

причем $u_{i}(\xi) \in\{\xi\} \cdot E_{k}[\xi], i=1,2$.

Из (6) следует: $x_{1}+u_{i} \cdot x_{2}+u_{i} \cdot x_{3}+\ldots+u_{i} \cdot x_{p+1} \in K(M), i=1,2$. Используя эти л.-а. функции, операции подстановки, переименования и отождествления переменных, получаем л.-а. функцию $f\left(x_{1}, x_{2}, x_{3}\right)=x_{1}+u_{1} \cdot x_{1}+u_{2} \cdot x_{2}-\left(u_{1}+u_{2}\right) x_{3}$. Применяя 
операцию обратной связи к единственной переменной функции $f\left(\nu_{0}, \nu_{0}^{\prime}, x\right)$, получим константу 0.

Далее используем (5). Подставляя константу 0 вместо переменных $x_{3}, x_{4}, \ldots, x_{p+1}$ сумматора $x_{1}+x_{2}+\ldots+x_{p+1}$, получим сумматор $x_{1}+x_{2}$. Для любого $\mu, \mu \in E_{k}^{\prime}(\xi)$, выполнено (6). Поэтому $\mu \cdot x=0+\mu \cdot x+\mu \cdot 0+\ldots+\mu \cdot 0 \in K(M)$. Значит, $\xi \cdot x \in K(M)$ и $a \cdot x \in K(M)$ для любого $a, a \in E_{k}$. Далее, $\left(\nu_{0}^{\prime}\right)^{-1}(x) \in K(M)$, поэтому $1=\left(\nu_{0}^{\prime}\right)^{-1}\left(\nu_{0}^{\prime}\right) \in K(M)$. Отсюда и из леммы 1 следует равенство $(2)$.

Теорема 2 доказана.

В соответствии с теоремой 2 проверка $K$-полноты конечной системы $M, M \subseteq \mathfrak{L}_{k}$, сводится к проверке свойств (3) и (4). Проверка свойства (3) не вызывает затруднений, поэтому в дальнейшем будет построен алгоритм проверки свойства (4).

Замыкание множества $M, M \subseteq E_{k}^{\prime}(\xi)$, по операциям сложения и умножения будем обозначать $S^{1}(M)$. В кольце $E_{k}^{\prime}(\xi)$ помимо операций сложения и умножения будем рассматривать также операцию "Об". Для элементов $\mu_{1}$ и $\mu_{2}$ из $E_{k}^{\prime}(\xi)$ значение Об $\left(\mu_{1}, \mu_{2}\right)$ определено в точности тогда, когда $\mu_{2}(0)=0$. Если последнее равенство спрведливо, то

$$
\text { Об }\left(\mu_{1}, \mu_{2}\right)=\frac{\mu_{1}}{1-\mu_{2}} \text {. }
$$

Замыкание множества $M, M \subseteq E_{k}^{\prime}(\xi)$, по операциям сложения, умножения и операции Об обозначаем $K^{1}(M)$.

В соответствии с [4] множество $M, M \subseteq E_{k}^{\prime}(\xi)$, называется $K^{1}$-замкнутым, если

$$
K^{1}(M)=E_{k}^{\prime}(\xi)
$$

Следуя рассуждениям, приведенным в [7], можно убедиться в справедливости включения

$$
U(K(M)) \subseteq K^{1}(U(M))
$$

Поэтому равенство

$$
K^{1}(U(M))=E_{k}^{\prime}(\xi)
$$

является необходимым условием $K$-полноты множества $M, M \subseteq \mathfrak{L}_{k}$. В дальнейшем будет найден алгоритм проверки $K^{1}$-полноты конечных подмножеств кольца $E_{k}^{\prime}(\xi)$, которое, что доказывается с использованием рассуждений из [7], изоморфно классу одноместных линейно-автоматных функций, сохраняющих нулевую последовательность.

\section{2. Класс одноместных линейно-автоматных функ- ций, сохраняющих нулевую последовательность}

Сначала рассмотрим свойства некоторых полей.

В конечном поле $E_{k}$,

$$
E_{k}=\left\{d_{0}+d_{1} z+\ldots+d_{m-1} z^{m-1} \mid d_{i} \in E_{p}, \quad i=0,1, \ldots, m-1\right\},
$$

введены операции сложения и умножения по модулю некоторого неприводимого над $E_{p}$ многочлена степени $m$.

Пусть $\eta \in E_{k}(\xi)$. Через $E_{p}(\eta)$ обозначим поле, получаемое простым трансцендентным расширением [1; стр. 141] поля $E_{p}$ элементом $\eta$. 
Пусть $M \subseteq E_{k}(\xi)$. Через $E_{p}(M)$ обозначим поле, получаемое расширением поля $E_{p}$ элементами множества $M$.

Рассмотрим поле $E_{k}(\xi)$. Если $M \subseteq E_{k}(\xi)$ и $M \backslash E_{k} \neq \varnothing$, то найдется $\eta, \eta \in$ $M \backslash E_{k}$, для которого имеем конечную башню полей: $E_{p}(\eta) \subset E_{k}(\eta) \subset E_{k}(\xi)$ (см. [5; стр. 185-187]).

Поле $E_{k}(\eta)$ - векторное пространство над полем $E_{p}(\eta)$ с базисом $\left\{z^{0}, z^{1}, \ldots, z^{m-1}\right\}$.

Обозначим через $s$ степень дроби $\eta$ по переменной $\xi$. Поле $E_{k}(\xi)-$ векторное пространство над полем $E_{k}(\eta)$ размерности $s$, с базисом $\left\{\xi^{0}, \xi^{1}, \ldots, \xi^{s-1}\right\}$.

Поэтому множество

$$
\left\{z^{i} \xi^{j} \mid i=0,1, \ldots, m-1, j=0,1, \ldots, s-1\right\}
$$

является базисом векторного пространства $E_{k}(\xi)$ над полем $E_{p}(\eta)$ [3; стр. 77].

Положим $M^{1}=M$ и $M^{i}=M^{i-1} * M \cup M^{i-1}$ для любого $i, i \in \mathbb{N}, i>1$.

Лемма 3. Поле $E_{p}(M)$ как векторное пространство над полем $E_{p}(\eta)$ порождается множеством $M^{\text {sm }}$.

Доказательство. Если для некоторого натурального $i$ справедливо

$$
\operatorname{dim}_{E_{p}(\eta)} M^{i}=\operatorname{dim}_{E_{p}(\eta)} M^{i+1},
$$

то для любого натурального $j, j \geqslant i$, имеем:

$$
\operatorname{dim}_{E_{p}(\eta)} M^{i}=\operatorname{dim}_{E_{p}(\eta)} M^{j} .
$$

Tак как $\operatorname{dim}_{E_{p}(\eta)} M^{i} \leqslant m s$, то

$$
\operatorname{dim}_{E_{p}(\eta)} M^{m s}=\operatorname{dim}_{E_{p}(\eta)} E_{p}(M) .
$$

Лемма доказана.

Следствие 1. Равенство

$$
E_{p}(M)=E_{k}(\xi)
$$

справедливо в точности тогда, когда

$$
\operatorname{dim}_{E_{p}(\eta)} M^{m s}=m s .
$$

Лемма 4. Пусть $M \subseteq E_{k}^{\prime}(\xi), E_{p}(M)=E_{k}(\xi)$. Тогда для некоторого $\mu$, $\mu \in E_{k}^{\prime}(\xi) \backslash\{0\}$, любого $a, a \in E_{k}$, имеет место включение

$$
\{a \cdot \mu, a \cdot \xi \mu\} \subset S^{1}(M) .
$$

Доказательство. Пусть $M \subseteq E_{k}^{\prime}(\xi), E_{p}(M)=E_{k}(\xi)$. Тогда в $E_{k}^{\prime}(\xi) \backslash\{0\}$ найдутся $\mu_{1}$ и $\mu_{2}$, что

$$
\left\{\mu_{1}, z \cdot \mu_{1}, \mu_{2}, \xi \cdot \mu_{2}\right\} \subset S^{1}(M) .
$$

Положим $\mu=\left(\mu_{1} \mu_{2}\right)^{m-1}$. Тогда для любого $i, i=0,1, \ldots, m-1$, справеливы включения

$$
z^{i} \cdot \mu \in S^{1}(M)
$$

и

$$
z^{i} \cdot \xi \cdot \mu \in S^{1}(M) .
$$

Отсюда, для любого $a, a \in E_{k}$, используя операцию сложения, получаем включение (12).

Лемма доказана. 
Используя рассуждения из [7], несложно доказать следующую лемму.

Лемма 5. Для любого $M, M \subseteq E_{k}^{\prime}(\xi)$, и для любого $\mu, \mu \in K^{1}(M)$, найдутся такие $\mu_{i}, \mu_{i} \in S^{1}(M), i=1,2$, что $\mu=$ Об $\left(\mu_{1}, \mu_{2}\right)$.

Пусть $\mu \in E_{k}^{\prime}(\xi), \mu=\frac{u}{v}, \operatorname{deg} u \leqslant \operatorname{deg} v$. Тогда для некоторых $s, a, a^{\prime}, b, b^{\prime}, u^{\prime}, v^{\prime}$, $s \in \mathbb{N}, \quad a, a^{\prime} \in E_{k}, \quad b, b^{\prime} \in E_{k} \backslash\{0\}, \quad u^{\prime}, v^{\prime} \in E_{k}[\xi], \quad \operatorname{deg} u^{\prime}<s-1, \operatorname{deg} v^{\prime}<s-1$, имеет место равенство

$$
\mu=\frac{a+\xi u^{\prime}+a^{\prime} \xi^{s}}{b+\xi v^{\prime}+b^{\prime} \xi^{s}}
$$

Через $\Psi_{0}(\mu)$ обозначим пару $\left(\frac{a}{b}, \frac{a^{\prime}}{b^{\prime}}\right)$ элементов поля $E_{k}$. Нетрудно видеть, что пара $\Psi_{0}(\mu)$ определяется по $\mu$ однозначно, потому что сохраняется после умножения числителя и знаменателя дроби $\mu$ на один и тот же, не делящийся на $\xi$, многочлен.

Если $M \subseteq E_{k}^{\prime}(\xi)$, то $\Psi_{0}(M)=\left\{\Psi_{0}(\mu) \mid \mu \in M\right\}$.

Положим

$$
M_{0}^{(1)}=\left\{\mu \mid \mu \in E_{k}^{\prime}(\xi), \quad \mu=\frac{u}{v}, \operatorname{deg} u \leqslant \operatorname{deg} v\right\} .
$$

Лемма 6. Пусть $M \subseteq M_{0}^{(1)}$. Соотношение

$$
K^{1}(M) \nsubseteq M_{0}^{(1)}
$$

выполнено в точности тогда, когда найдется такое $b, b \in E_{k} \backslash\{0\}$, что

$$
(0, b) \in \Psi_{0}\left(S^{1}(M)\right) .
$$

Доказательство. Если $M \subseteq M_{0}^{(1)}$, то $S^{1}(M) \subseteq M_{0}^{(1)}$. Поэтому из (13) и леммы 5 вытекает, что найдутся такие $\mu_{j}, \mu_{j} \in S^{1}(M), j=1,2$, что Об $\left(\mu_{1}, \mu_{2}\right) \notin M_{0}^{(1)}$. Тогда для некоторого $b, b \in E_{k} \backslash\{0\}$, выполнено: $\Psi_{0}\left(\mu_{2}\right)=(0, b)$, откуда вытекает (14).

Обратно, пусть выполнено (14) и $\mu, \mu \in S^{1}(M)$, таково, что для некоторого $b$, $b \in E_{k} \backslash\{0\}$, имеем $\Psi_{0}(\mu)=(0, b)$. Тогда для дроби $\mu^{\prime}, \mu^{\prime}=\frac{u^{\prime}}{v^{\prime}}, \mu^{\prime}=$ Об $\left(\mu, \mu^{k-1}\right)$, имеем $\operatorname{deg} u^{\prime}>\operatorname{deg} v^{\prime}$. Поэтому (13) выполнено.

Лемма доказана.

На множестве $E_{k}^{2}$ пар чисел поля $E_{k}$ будем рассматривать операции сложения и умножения, которые определяются покомпонентно:

$$
\begin{gathered}
\left(a_{1}, b_{1}\right)+\left(a_{2}, b_{2}\right)=\left(a_{1}+a_{2}, b_{1}+b_{2}\right), \\
\left(a_{1}, b_{1}\right) \cdot\left(a_{2}, b_{2}\right)=\left(a_{1} a_{2}, b_{1} b_{2}\right) .
\end{gathered}
$$

Замыкание множества $M, M \subseteq E_{k}^{2}$, по операциям сложения и умножения обозначим $S(M)$.

Нетрудно видеть, что $\Psi_{0}\left(S^{1}(M)\right)=S\left(\Psi_{0}(M)\right)$.

Учитывая, что множество, состоящее из всех неприводимых в $E_{k}[\xi]$ приведенных многочленов, счетно [6], упорядочим эти многочлены некоторым образом: $p_{1}(\xi), p_{2}(\xi), \ldots$ так, что $p_{1}(\xi)=\xi$.

Положим

$$
M_{i}^{(1)}=\left\{\mu \mid \mu \in E_{k}^{\prime}(\xi), \quad \mu=\frac{u}{v},\left(v, p_{i}\right)=1\right\},
$$

$i=2,3, \ldots$ Пусть $\mu \in M_{i}^{(1)}$. Тогда в $E_{k}[\xi]$ найдутся такие многочлены $u^{\prime}, u^{\prime \prime}, v^{\prime}$, что $\operatorname{deg} u^{\prime} \leqslant \operatorname{deg} p_{i}$ и

$$
\mu=\frac{u^{\prime}+\xi p_{i} u^{\prime \prime}}{1-\xi p_{i} v^{\prime}}
$$


Через $\Psi_{i}(\mu)$ обозначим пару $\left(\mu(0), u^{\prime}\right)$.

Нетрудно видеть, что $\mu(0)=u^{\prime}(0)$.

Лемма 7. Пусть для некоторого $i, i \in\{2,3, \ldots\}$, имеет место включение $M \subseteq$ $M_{i}^{(1)}$. Соотношение

$$
K^{1}(M) \nsubseteq M_{i}^{(1)}
$$

выполнено в точности тогда, когда в $\Psi_{i}\left(S^{1}(M)\right)$ найдется пара $(0, v)$ с $v \neq 0$.

Доказательство. Пусть $M \subseteq M_{i}^{(1)}$ для некоторого $i, i \in \mathbb{N}$, и выполнено (15). Тогда по лемме 5 найдутся такие $\mu_{j}, \mu_{j} \in S^{1}(M)$,

$$
\mu_{j}=\frac{u_{j}^{\prime}+\xi p_{i} u_{j}^{\prime \prime}}{1-\xi p_{i} v_{j}^{\prime}}
$$

$\operatorname{deg} u_{j}^{\prime} \leqslant \operatorname{deg} p_{i}, j=1,2$, что Об $\left(\mu_{1}, \mu_{2}\right) \notin M_{i}^{(1)}$. Равенство нулю числа $u_{2}^{\prime}(0)$ следует из возможности применения операции Об к паре $\mu_{1}, \mu_{2}$. Неравенство $u_{2}^{\prime} \neq 0$ получаем ввиду того, что $p_{i}$ делит многочлен $1-\xi p_{i} v_{2}^{\prime}-u_{2}^{\prime}-\xi p_{i} u_{2}^{\prime \prime}$.

В обратную сторону. Пусть $M \subseteq M_{i}^{(1)}$ и найдется такая $\mu, \mu \in S^{1}(M)$, что $\Psi_{i}(\mu)$ имеет нулевую первую компоненту и ненулевую вторую. Тогда для некоторого натурального числа $T$ числитель дроби $1-\mu^{T}$, предствленной в несократимом виде, делится на $p_{i}$. Поэтому Об $\left(\mu, \mu^{T}\right) \notin M_{i}^{(1)}$.

Лемма доказана.

Для некоторого целого неотрицательного числа $\tau$ множество $M, M \subseteq E_{k}^{\prime}(\xi)$, назовем $\tau$-предельным, если

$$
\xi^{\tau} E_{k}^{\prime}(\xi) \subseteq K^{1}(M) .
$$

Множество $M$ назовем предельным, если найдется $\tau$, для которого оно является $\tau$-предельным.

Лемма 8. Пусть $M \subseteq E_{k}^{\prime}(\xi)$. Множество $M$ являетя предельным в точности тогда, когда имеет место условие (10), а также для любого $i, i=0,2,3, \ldots$, из $M \subseteq M_{i}^{(1)}$ следует (15).

Доказательство. Пусть множество $M$ является предельным. Тогда для некоторого натурального $\tau$ выполнено включение (16). Поэтому множество $M^{\prime}$,

$$
M^{\prime}=\left\{\xi^{\tau}, a \cdot \xi^{\tau}, \xi^{\tau+1} \mid a \in E_{k}\right\}
$$

содержится в $K^{1}(M)$.

Нетрудно видеть, что множество $M^{\prime \prime}$,

$$
M^{\prime \prime}=\left\{a, \quad \xi \mid a \in E_{k}\right\},
$$

содержится в $E_{p}\left(M^{\prime}\right)$. Далее из соотношения

$$
E_{k}(\xi)=E_{p}\left(M^{\prime \prime}\right) \subseteq E_{p}(M) \subseteq E_{k}(\xi) .
$$

получаем равенство (10).

Для любого целого числа $i, i \in\{0,2,3, \ldots\}$, имеем:

$$
\xi^{\tau} E_{k}^{\prime}(\xi) \nsubseteq M_{i}^{(1)}
$$


откуда с учетом (16) получаем (15).

В обратную сторону. Пусть для множества $M, M \subseteq E_{k}^{\prime}(\xi)$, выполнены (10) и (15) для $i=0,2,3, \ldots$.

Тогда из леммы 4 и соотношения (13) для некоторого $\tilde{\mu}, \tilde{\mu}=\frac{\tilde{u}}{\tilde{v}}, \operatorname{deg}(\tilde{u}) \geqslant \operatorname{deg}(\tilde{v})$, и любого $a, a \in E_{k}$, имеем:

$$
\{a \tilde{\mu}, a \xi \tilde{\mu}\} \subset K^{1}(M) .
$$

Поэтому, используя рассуждения из доказательства леммы 9 работы [7], заключаем, что существуют такие целые неотрицательные числа $s_{1}$ и $s_{2}$, что для любых таких целых неотрицательных чисел $i$ и $j$, что $i \geqslant s_{1}, j \geqslant s_{2}$, имеем

$$
\xi^{i} \tilde{\mu}^{j} \in K^{1}(M)
$$

Поэтому для любого $a, a \in E_{k}$, и любых таких целых неотрицательных чисел $i$ и $j$, что $i \geqslant s_{1}, j \geqslant s_{2}+1$, получаем:

$$
a \xi^{i} \tilde{\mu}^{j} \subseteq K^{1}(M)
$$

Отсюда и из условий (15) с использованием рассуждений из доказательства той же леммы работы [7] заключаем, что $M$ - предельное множество.

Лемма доказана.

Положим

$$
M_{1}^{(1)}=\left\{\mu \mid \mu \in E_{k}^{\prime}(\xi), \mu(\xi)-\mu(0) \in \xi^{2} \cdot E_{k}^{\prime}(\xi)\right\} .
$$

Имеем разложение числа $m$ в произведение простых чисел $q_{1}, q_{2}, \ldots, q_{s}$ :

$$
m=q_{1}^{r_{1}} \cdot q_{2}^{r_{2}} \ldots q_{l}^{r_{l}}
$$

$q_{s} \neq q_{s^{\prime}}$ при $s \neq s^{\prime}$.

Обозначим через $k_{s}$ число $p^{m / q_{s}}, s=1,2, \ldots, l$. Для любого $s, s=1,2, \ldots, l$, в поле $E_{k}$ существует единственное подполе $E_{k_{s}}$, содержащее $k_{s}$ элементов [6].

Для каждого $s, s \in\{1,2, \ldots, l\}$, следующим образом определим множество $P_{s}^{(1)}$ :

$$
P_{s}^{(1)}=\left\{\mu(\xi) \mid \mu \in E_{k}^{\prime}(\xi), \mu(0) \in E_{k_{s}}\right\} .
$$

Теорема 3. Пусть $M \subseteq E_{k}^{\prime}(\xi)$. Равенство

$$
K^{1}(M)=E_{k}^{\prime}(\xi)
$$

выполнено в точности тогда, когда имеет место условие (10), для любого $i, i=$ $0,1, \ldots$, условие (15), а также для любого $s, s \in\{1,2, \ldots, l\}$, следующее условие:

$$
M \nsubseteq P_{s}^{(1)} \text {. }
$$

Доказательство. Пусть имеет место (18).

Через а обозначим какой-либо примитивный элемент поля $E_{k}$. Из $(18)$ для множества $M_{0}, M_{0}=\{\xi, a\}$, имеет место включение $M_{0} \subset K^{1}(M)$. Учитывая, что $E_{p}\left(M_{0}\right)=E_{k}(\xi)$, получаем $(10)$.

Справедливость соотношений (15) вытекает из неравенств

$$
M_{i}^{(1)} \neq E_{k}^{\prime}(\xi), i=0,1, \ldots
$$


Свойство (19) множества $M$ следует из $K^{1}$-замкнутости множеств $P_{s}^{(1)}$ и неравенств $P_{s}^{(1)} \neq E_{k}^{\prime}(\xi), s=1,2, \ldots, l$.

В обратную сторону. Пусть для множества $M, M \subseteq E_{k}^{\prime}(\xi)$, выполнены соотношения (10), (15) при $i=0,1, \ldots,(19)$ при $s=1,2, \ldots, l$.

Для числа $m$ имеем разложение (17). Через $\mu_{s}$ обозначим элемент множества $M$, не содержащийся в $P_{s}^{(1)}, s=1,2, \ldots, l$. Порядок числа $b_{s}, b_{s}=\mu_{s}(0)$, равен $p^{c_{s} \cdot q_{s}^{r_{s}}}-1$ для некоторого делителя $c_{s}$ числа $\frac{m}{q_{s}^{r}}$. Положим $c_{s}^{\prime}=\sum_{i=0}^{c_{s}-1} p^{i q_{s}^{r_{s}}}$ Порядок числа $d_{s}$, $d_{s}=b_{s}^{c_{s}^{\prime}}$, равен $p^{q_{s}^{r_{s}}}-1$

Отсюда и из [6] следует, что для любого $s^{\prime}, s^{\prime} \in\{1,2, \ldots, l\} \backslash\{s\}$, выполнены соотношения $d_{s} \in E_{k_{s^{\prime}}}$ и $d_{s} \notin E_{k_{s}}$.

Поэтому число $a_{0}, a_{0}=d_{1} \cdot d_{2} \cdot \ldots \cdot d_{l}$, не содержится в $E_{k_{s}}$ для любого $s, s=$ $1,2, \ldots, l$, то есть является примитивным элементом поля $E_{k}$.

Из приведенных рассуждений следует, что для любого $a, a \in E_{k}$, используя операцию умножения, из элементов множества $M$ можно получить такую дробь $\mu_{a}$, что $\mu_{a}(0)=a$.

Далее, ввиду соотношения $M \nsubseteq M_{1}^{(1)}$, в $M$ содержится такая дробь $\mu^{\prime}$, что для некоторых $b_{0}^{\prime}, b_{1}^{\prime}, \mu^{\prime \prime}, \quad b_{0}^{\prime} \in E_{k}, \quad b_{1}^{\prime} \in E_{k} \backslash\{0\}, \quad \mu^{\prime \prime} \in E_{k}^{\prime}(\xi)$ выполнено

$$
\mu^{\prime}=b_{0}^{\prime}+b_{1}^{\prime} \xi\left(1+\xi \mu^{\prime \prime}\right) \text {. }
$$

Используя дробь $\mu^{\prime}$, а также дроби $\mu_{a}, a \in E_{k}$, индукцией по $s, s \geqslant 1$, нетрудно показать, что для любого многочлена $u, u \in E_{k}[\xi], \operatorname{deg} u<s$, найдется такая дробь $\mu_{u}$ из $E_{k}^{\prime}(\xi)$, что

$$
u+\xi^{s} \mu_{u} \in K^{1}(M) .
$$

Из этого и из леммы 8 следует, что $E_{k}[\xi] \subset K^{1}(M)$, откуда вытекает равенство (18).

Теорема доказана.

\section{3. Редукция}

Лемма 9. Пусть

$$
h=\sum_{i=1}^{n} \mu_{i} \sum_{j=1}^{p} x_{i, j}+\sum_{i=1}^{n} \mu_{i} \sum_{j=1}^{p} x_{i, j}^{\prime}+\mu^{\prime} x^{\prime}+\mu_{0}
$$

$u$

$$
M=\left\{\mu_{i} \mid i=1,2, \ldots, n\right\} .
$$

Тогда для любой $\mu, \mu \in S^{1}(M)$, найдутся такие $\mu^{\prime \prime} u \nu$, что

$$
\mu x_{1}+\mu x_{2}+\mu^{\prime \prime} x+\nu \in S(\{h\}) .
$$

Доказательство. Пусть имеют место равенства (20) и (21). Для л.-а. функции $h$ справедливо равенство

$$
\begin{aligned}
h= & h\left(x_{1,1}, x_{1,2}, \ldots x_{1, p}, x_{2,1}, x_{2,2}, \ldots x_{2, p}, \ldots, x_{n, 1}, x_{n, 2}, \ldots x_{n, p}\right. \\
& \left.x_{1,1}^{\prime}, x_{1,2}^{\prime}, \ldots x_{1, p}^{\prime}, x_{2,1}^{\prime}, x_{2,2}^{\prime}, \ldots x_{2, p}^{\prime}, \ldots, x_{n, 1}^{\prime}, x_{n, 2}^{\prime}, \ldots x_{n, p}^{\prime}, x^{\prime}\right) .
\end{aligned}
$$


Если $\mu \in S^{1}(M)$, то $\mu$ является многочленом от $n$ переменных $\mu_{1}, \mu_{2}, \ldots, \mu_{n}$ степени, не меньшей 1.

Доказательство леммы проведем индукцией по степени $\operatorname{deg}_{M}(\mu)$ этого многочлена.

Если $\operatorname{deg}_{M} \mu=1$, то найдутся такие числа $d_{1}, d_{2}, \ldots, d_{n}$ из $E_{p}$, что

$$
\mu=\sum_{i=1}^{n} d_{i} \mu_{i}
$$

Положим

$$
\begin{aligned}
h^{\prime}\left(x_{1}, x_{2}, x\right)= & h(\underbrace{x_{1}, \ldots x_{1}}_{d_{1}}, \underbrace{x, \ldots, x}_{p-d_{1}}, \ldots, \underbrace{x_{1}, \ldots x_{1}}_{d_{n}}, \underbrace{x, \ldots, x}_{p-d_{n}}, \\
& \underbrace{x_{2}, \ldots x_{2}}_{d_{1}}, \underbrace{x, \ldots, x}_{p-d_{1}}, \ldots, \underbrace{x_{2}, \ldots x_{2}}_{d_{n}}, \underbrace{x, \ldots, x}_{p-d_{n}}, x) .
\end{aligned}
$$

Тогда для некоторых $\mu^{\prime \prime}$ и $\nu$ выполнено (22).

Предположим, что для некоторого натурального числа $r$ и любого $\mu, \mu \in S^{1}(M)$, $\operatorname{deg}_{M} \mu \leqslant r$, найдутся такие $\mu^{\prime \prime}$ и $\nu$, что выполнено (22). Пусть $\mu, \mu \in S^{1}(M)$, таково, что $\operatorname{deg}_{M} \mu=r+1$.

Тогда найдутся такие числа $d_{j}, d_{j} \in E_{p}, j=1,2, \ldots, n$, и одноместные сохраняющие нулевую последовательность л.-а. функции $\mu_{j}^{\prime}, j=1,2, \ldots, n$, что $\mu_{j}^{\prime} \in S^{1}(M)$, $\operatorname{deg}_{M} \mu_{j}^{\prime} \leqslant r, j=1,2, \ldots, n$, и

$$
\mu=\sum_{j=1}^{n} d_{j} \mu_{j}+\sum_{j=1}^{n} \mu_{j} \mu_{j}^{\prime} .
$$

Согласно предположению индукции, в $S(M)$ содержатся л.-а. функции $\tilde{h}_{j}\left(x, x_{1}\right)$,

$$
\tilde{h}_{j}\left(x, x_{1}\right)=\mu_{j}^{\prime} x+\mu_{j}^{\prime} x_{1}+\mu_{j}^{\prime \prime} x_{1}+\nu_{j} .
$$

Пусть

$$
\begin{aligned}
& h_{j}^{\prime}\left(x^{\prime}, x\right)=\left\{\begin{array}{ccc}
\tilde{h}_{j}\left(x^{\prime}, x\right), & \text { если } & \tilde{h}_{j}\left(x^{\prime}, x\right) \neq 0, \\
x, & \text { если } & \tilde{h}_{j}\left(x^{\prime}, x\right)=0,
\end{array}\right. \\
& \tilde{h}\left(x_{1}, x_{2}, x\right)= h(\underbrace{x_{1}, \ldots x_{1}}_{d_{1}}, h_{1}^{\prime}\left(x_{1}, x\right), \underbrace{x, \ldots, x}_{p-d_{1}-1}, \ldots, \\
& \underbrace{x_{1}, \ldots x_{1}}_{d_{n}}, h_{n}^{\prime}\left(x_{1}, x\right), \underbrace{x, \ldots, x}_{p-d_{n}-1}, \\
& \underbrace{x_{2}, \ldots x_{2}}_{d_{1}}, h_{1}^{\prime}\left(x_{2}, x\right), \underbrace{x, \ldots, x}_{p-d_{1}-1}, \ldots, \\
&\underbrace{x_{2}, \ldots x_{2}}_{2}, h_{n}^{\prime}\left(x_{2}, x\right), \underbrace{x, \ldots, x}_{p-d_{n}-1}, x) .
\end{aligned}
$$

Тогда для некоторых $\mu^{\prime \prime}$ и $\nu$ выполнено (22). Лемма доказана. 
В дальнейшем используем следующие множества одноместных л.-а. функций, сохраняющих нулевую последовательность:

$$
\begin{gathered}
W_{i}^{(1)}=\left\{\mu \mid \mu \in M_{i}^{(1)}, \text { для некоторого } b, b \neq 0, \Psi_{i}(\mu)=(0, b)\right\}, \\
i=0,2,3, \ldots
\end{gathered}
$$

Лемма 10. Для любого $\mu, \mu \in E_{k}^{\prime}(\xi)$, любого иелого неотрицательного $\tau$ и любого $i, i \in\{0,2,3, \ldots\}$, справедливы соотношения

$$
\begin{aligned}
& \mu \in M_{i}^{(1)} \Leftrightarrow \mu^{p^{\tau}} \in M_{i}^{(1)}, \\
& \mu \in W_{i}^{(1)} \Leftrightarrow \mu^{p^{\tau}} \in W_{i}^{(1)} .
\end{aligned}
$$

Теорема 4. Пусть для множества $M, M \subseteq \mathfrak{L}_{k}$, и любого $\Theta, \Theta \in J_{k}^{\prime}$, выполнено (3). Равенство (4) справедливо тогда и только тогда, когда $M$ обладает следующими свойствами:

$$
\begin{gathered}
E_{p}(U(M))=E_{k}(\xi) ; \\
\forall s, s=1,2, \ldots, l, \quad U(M) \nsubseteq P_{s}^{(1)} ; \\
\forall j, j=0,1,2, \ldots, \quad U(K(M)) \nsubseteq M_{j}^{(1)} .
\end{gathered}
$$

Доказательство. Пусть для множества $M, M \subseteq \mathfrak{L}_{k}$, выполнено (4). Тогда из (8) получаем равенство $K^{1}(U(M))=E_{k}^{\prime}(\xi)$. Поэтому $E_{k} \cup\{\xi\} \subset K^{1}(U(M))$. Отсюда и из включения $K^{1}(U(M)) \subseteq E_{p}(U(M))$ получаем (23). Свойство (24) вытекает из $K^{1}$-замкнутости собственных подмножеств $P_{s}^{(1)}, s=1,2, \ldots, l$, множества $\mathfrak{L}_{k}$, свойство $(25)$ - из неравенств $M_{j}^{(1)} \neq E_{k}^{\prime}(\xi), i=0,1,2, \ldots$

В обратную сторону. Пусть для $M, M \subseteq \mathfrak{L}_{k}$, выполнены (3), (23), (24) и (25). Докажем, что выполнено (5).

Из функции $f\left(x_{1}, x_{2}, \ldots, x_{n}\right), f \in M \backslash V_{1}$, с испольованием операций суперпозиции можно получить л.-а. функцию $g\left(x_{1}, x_{2}, x\right)$,

$$
g\left(x_{1}, x_{2}, x\right)=\mu_{1} \cdot x_{1}+\mu_{1} \cdot x_{2}+\mu^{\prime} \cdot x+\mu_{0},
$$

где $\mu_{1}(0)=1$. Если $\mu_{1}=1$, то

$$
x_{1}+x_{2}+\ldots+x_{p+1}=g\left(x_{1}, g\left(x_{2}, \ldots g\left(x_{p}, x_{p+1}, x\right) \ldots, x\right), x\right) .
$$

В противном случае одноместная л.-а. функция $\mu, \mu=\mu_{1}^{2}-\mu_{1}$, не содержится лишь в конечном множестве $\Omega$ классов системы $\left\{W_{i}^{(1)} \mid i=0,2,3, \ldots\right\}$. Положим

$$
\Omega^{\prime}=\left\{j \mid j \in \Omega, \quad \mu \in M_{j}^{(1)}\right\} \cup\{1\} .
$$

Согласно предположению, найдутся такие конечные множества $M_{l}^{\prime}, l=1,2,3$, что

$$
\begin{gathered}
M_{1}^{\prime} \subseteq U(M), \quad E_{p}\left(M_{1}^{\prime}\right)=E_{k}(\xi), \\
M_{2}^{\prime} \subseteq U(M), \quad \forall s, s=1,2, \ldots, l, \quad M_{2}^{\prime} \nsubseteq P_{s}^{(1)}, \\
M_{3}^{\prime} \subset K(U(M)), \quad \forall j, j \in \Omega^{\prime}, \quad M_{3}^{\prime} \nsubseteq M_{j}^{(1)} .
\end{gathered}
$$

Через $r$ обозначим число $1+\sum_{i=1}^{3}\left|M_{i}^{\prime}\right|$. Выберем натуральное число $\tau$, так, что $p^{\tau-1} \geqslant 2 r$. 
Положим

$$
\begin{gathered}
M_{1}^{\prime \prime}=\left\{\mu_{1}^{p^{\tau}}\right\} \cup\left\{\mu^{\prime} \mu_{1}^{p^{\tau}} \mid \mu^{\prime} \in M_{1}^{\prime}\right\}, \\
M_{2}^{\prime \prime}=\left\{\mu^{\prime} \mu_{1}^{p^{\tau}} \mid \mu^{\prime} \in M_{2}^{\prime}\right\} .
\end{gathered}
$$

Нетрудно видеть, что

$$
E_{p}\left(M_{1}^{\prime \prime}\right)=E_{k}(\xi)
$$

и

$$
\forall s, s=1,2, \ldots, l, \quad M_{2}^{\prime \prime} \nsubseteq P_{s}^{(1)} .
$$

Для каждого $\mu^{\prime} \in M_{3}^{\prime}$ найдется такое натуральное число $T\left(\mu^{\prime}\right)$, что множество

$$
M_{3}^{\prime \prime}=\left\{\left(\mu^{\prime}\right)^{T\left(\mu^{\prime}\right)} \mu_{1}^{p^{\tau}} \mid \mu^{\prime} \in M_{3}^{\prime}\right\}
$$

не содержится ни в одном из классов $M_{j}^{(1)}, j \in \Omega^{\prime}$.

Пусть

$$
M^{\prime}=\bigcup_{l=1}^{3} M_{l}^{\prime \prime} .
$$

Из свойств множества $M^{\prime}$, леммы 10 и теоремы 3 следует, что $1 \in K\left(M^{\prime}\right)$. Поэтому из леммы 5 вытекает, что для некоторых $\mu_{i}^{*}, \mu_{i}^{*} \in S\left(M^{\prime}\right), i=1,2$, имеет место равенство $1=\frac{\mu_{1}^{*}}{1-\mu_{2}^{*}}$, откуда $1=\mu_{1}^{*}+\mu_{2}^{*}$, то есть

$$
1 \in S^{1}\left(M^{\prime}\right) .
$$

Пусть

$$
M^{\prime}=\left\{\mu_{j}^{\prime} \mid j=1,2,3, \ldots, r\right\} .
$$

Нетрудно видеть, что л.-а. функция $h$,

$$
h=\sum_{i=1}^{r} \mu_{i}^{\prime} \sum_{j=1}^{p} x_{i, j}+\sum_{i=1}^{r} \mu_{i}^{\prime} \sum_{j=1}^{p} x_{i, j}^{\prime}+\mu^{\prime \prime} x+\mu_{0}^{\prime \prime},
$$

содержится в $K(M)$.

С учетом (26) из леммы 9 следует, что для некоторых $\mu^{*}$ и $\mu_{0}^{*}$ из $E_{k}^{\prime}(\xi)$

$$
x_{1}+x_{2}+\mu^{*} x+\mu_{0}^{*} \in S(\{h\}),
$$

откуда, как было показано выше, следует (5).

Равенство (4) несложно получить, используя включение (5) и теорему 3.

Теорема доказана.

Переменная $x_{i}$ л.-а. функции $f\left(x_{1}, x_{2}, \ldots, x_{n}\right)$, задаваемой равенством $(1)$, называется существенной, если $\mu_{i} \neq 0$. Если $\mu_{i}(0) \neq 0$, то переменная $x_{i}$ называется непосредственной.

Введем некоторые классы л.-а. функций:

$$
\begin{gathered}
\tilde{M}_{0}^{(1)}=\left\{\mu \mid \mu \in E_{k}^{\prime}(\xi), \quad \mu=\frac{u}{v}, \operatorname{deg} u<\operatorname{deg} v\right\}, \\
\tilde{M}_{i}^{(1)}=\left\{\mu \mid \mu \in E_{k}^{\prime}(\xi), \quad \mu=\frac{u}{v}, \quad(u, v)=1, p_{i} \text { делит } u\right\}, i=2,3, \ldots,
\end{gathered}
$$




$$
\begin{aligned}
R_{i}^{\mathrm{c}}= & \left\{f \mid f \in \mathfrak{L}_{k}, \text { выполнено }(1), \forall j, j=1,2, \ldots, n, \text { если } x_{j}-\right. \\
& \text { единственная существенная переменная функции } f, \text { то } \\
& \left.\mu_{j} \in M_{i}^{(1)}, \text { в противном случае: } \mu_{j} \in \tilde{M}_{i}^{(1)}\right\}, \\
R_{i}^{\mathrm{H}}= & \left\{f \mid f \in \mathfrak{L}_{k}, \text { выполнено }(1), \forall j, j=1,2, \ldots, n, \text { если } x_{j}-\right. \\
& \text { единственная непосредственная переменная функции } f, \\
& \text { то } \left.\mu_{j} \in M_{i}^{(1)}, \text { в противном случае: } \mu_{j} \in \tilde{M}_{i}^{(1)}\right\}, i=0,2,3, \ldots
\end{aligned}
$$

Положим

$$
J_{k}^{\prime \prime}=\left\{R_{i}^{\mathrm{c}}, R_{i}^{\mathrm{H}} \mid i=0,2,3, \ldots\right\} .
$$

Следующее утверждение приведем без доказательства.

Теорема 5. Каждый элемент множества $J_{k}^{\prime \prime}$ является K-замкнутым классом в $\mathfrak{L}_{k}$, не совпадаюшим с $\mathfrak{L}_{k}$.

Обозначим через $J_{k}$ следующее множество $K$-замкнутых классов:

$$
J_{k}=J_{k}^{\prime} \cup J_{k}^{\prime \prime} .
$$

Лемма 11. Пустъ $i \in\{0,2,3, \ldots\}, M \subseteq \mathfrak{L}_{k}, U(M) \subseteq M_{i}^{(1)}$,

$$
\forall \Theta, \Theta \in J_{k}^{\prime} \cup\left\{R_{i}^{c}, R_{i}^{H}\right\} \text {, имеет место } M \nsubseteq \Theta \text {. }
$$

Соотношение

$$
U(K(M)) \nsubseteq M_{i}^{(1)}
$$

выполнено в точности тогда, когда

$$
S^{1}(U(M)) \cap W_{i}^{(1)} \neq \varnothing .
$$

Для доказательства этой леммы нам понадобится понятие $i$-сумматора. Л.-а. функция $g\left(x_{1}, x_{2}\right), g\left(x_{1}, x_{2}\right)=\mu_{1} x_{1}+\mu_{2} x_{2}+\mu_{0}$, называется $i$-сумматором,$i \in$ $\{0,2,3, \ldots\}$, если $\mu_{i}(0)=1, \mu_{i}-\mu_{i}(0) \in \tilde{M}_{i}^{(1)}, i=1,2$.

Замечание 1. Если л.-а. функция $f$,

$$
f\left(x_{1}, x_{2}, x\right)=\mu_{1} x_{1}+\mu_{2} x_{2}+\mu,
$$

такова, что $x_{1}$ и $x_{2}$ - непосредственные переменные и $\mu_{i} \notin \tilde{M}_{i}^{(1)}, i=1,2$, а $\nu \in E_{k}^{\prime}$, то $S(\{f, \nu\})$ содержит $i$-сумматор.

Доказательство. Рассмотрим множество $M$ л.-а. функций, не содержащееся для некоторого целого неотрицательного числа $i$, не равного 1 , ни в одном из замкнутых классов множетва $J_{k}^{\prime} \cup\left\{R_{i}^{\mathrm{C}}, R_{i}^{\mathrm{H}}\right\}$, и такое, что $U(M) \subseteq M_{i}^{(1)}$.

Если имеет место (28), то из (8) с учетом лемм 6 и 7 заключаем, что неравенство (29) выполняется.

В обратную сторону: пусть имеет место (29), но (28) не выполнено. Сначала покажем, что в $K(M)$ содержится $i$-сумматор.

Рассмотрим функцию $g\left(x_{1}, x_{2}, \ldots, x_{m}\right) \in M \backslash R_{i}^{\mathrm{c}}$,

$$
g\left(x_{1}, x_{2}, \ldots, x_{m}\right)=\sum_{j=1}^{m} \mu_{j}^{\prime} x_{j}+\mu^{\prime} .
$$


Без ограничения общности предположим, что $\mu_{1}^{\prime} \notin \tilde{M}_{i}^{(1)}$ и $\mu_{2}^{\prime} \neq 0$. По лемме 2 в $K(M)$ содержится некоторая константа $\nu$. Тогда для л.-а. функции $g^{\prime}$,

$$
g^{\prime}\left(x_{1}, x_{2}\right)=g\left(x_{1}, x_{2}, \nu, \nu \ldots, \nu\right),
$$

найдется такое $\mu_{0}^{\prime}, \mu_{0}^{\prime} \in E_{k}^{\prime}(\xi)$, что

$$
g^{\prime}\left(x_{1}, x_{2}\right)=\mu_{1}^{\prime} x_{1}+\mu_{2}^{\prime} x_{2}+\mu_{0}^{\prime} .
$$

Случай 1. Если $\mu_{1}^{\prime}(0)=0$, то найдутся такие натуральные числа $T$ и $\tau$, что

$$
\frac{\mu_{2}^{\prime}}{1-\left(\mu_{1}^{\prime}\right)^{T \cdot p^{\tau}}} \notin M_{i}^{(1)},
$$

то есть выполнено (28), что противоречит предположению.

Случай 2. $\mu_{1}^{\prime}(0) \neq 0$. Тогда рассмотрим следующие случаи.

Случай 2.1. $\mu_{2}^{\prime} \notin \tilde{M}_{i}^{(1)}$. Тогда при $\mu_{2}^{\prime}(0)=0$, как и в случае 1 , получим противоречие с предположением о не выполнимости соотношения $(28)$, а при $\mu_{2}^{\prime}(0) \neq 0$, учитывая последнее замечание, заключаем, что $K(M)$ содержит $i$-сумматор.

Случай 2.2. Пусть $\mu_{2}^{\prime} \in \tilde{M}_{i}^{(1)}$. Рассматриваем:

Случай 2.2.1. $\mu_{2}^{\prime}(0) \neq 0$. Тогда, используя операции суперпозиции, из функции $g^{\prime}\left(x_{1}, x_{2}\right)$ и константы $\nu$ получим функцию $g^{\prime \prime}\left(x_{1}, x_{2}\right)$,

$$
\begin{gathered}
\left.g^{\prime \prime}=g^{\prime}(\underbrace{g^{\prime}\left(\ldots g ^ { \prime } \left(g^{\prime}\right.\right.}_{k-2}\left(x_{1}, \nu\right), \nu) \ldots,\right), \underbrace{g^{\prime}\left(\nu, g^{\prime}\left(\nu, \ldots g^{\prime}\right.\right.}_{k-2}\left(\nu, x_{2}\right) \ldots))), \\
g^{\prime \prime}\left(x_{1}, x_{2}\right)=\mu_{1}^{\prime \prime} x_{1}+\mu_{2}^{\prime \prime} x_{2}+\mu_{0}^{\prime \prime},
\end{gathered}
$$

где $\mu_{i}^{\prime \prime}=\left(\mu_{i}^{\prime}\right)^{k-1}, i=1,2$.

Имеем: $\mu_{i}^{\prime \prime}(0)=1, i=1,2, \mu_{1}^{\prime \prime} \notin \tilde{M}_{i}^{(1)}, \mu_{2}^{\prime \prime} \in \tilde{M}_{i}^{(1)}$.

В $K(M)$ содержится л.-а. функция $\tilde{g}\left(x_{1}, x_{2}\right)$,

$$
\tilde{g}=\underbrace{g^{\prime \prime}\left(x_{1}, g^{\prime \prime}\left(x_{1}, \ldots, g^{\prime \prime}\right.\right.}_{p \text { раз }}\left(x_{1}, x_{2}\right) \ldots)),
$$

Имеем

$$
\tilde{g}\left(x_{1}, x_{2}, x_{3}\right)=\tilde{\mu}_{1} x_{1}+\tilde{\mu}_{2} x_{2}+\tilde{\mu}_{0},
$$

причем $\tilde{\mu}_{1}=\sum_{j=0}^{p-1} \mu_{1}^{\prime \prime}\left(\mu_{2}^{\prime \prime}\right)^{j}, \tilde{\mu}_{2}=\left(\mu_{2}^{\prime \prime}\right)^{p}$, причем $\tilde{\mu}_{1}(0)=0, \tilde{\mu}_{1} \notin \tilde{M}_{i}^{(1)}, \tilde{\mu}_{2} \neq 0$. Как показано в случае 1 , используя л.-а. функцию $\tilde{g}$ и операции композиции, можно получить такую л.-.а. функцию $\hat{g}$, что $U(\hat{g}) \notin M_{i}^{(1)}$, что противоречит предположению.

Случай 2.2.2. $\mu_{2}^{\prime}(0)=0$. Тогда $x_{1}$ - единственная непосредственная переменная л.-а. функции

$$
g^{\prime}=g^{\prime}\left(x_{1}, x_{2}\right) .
$$

По условию найдется л.-а. функция $f, f \in M \backslash R_{i}^{\mathrm{H}}$, для которой выполнено (1). Пусть $x_{1}$ не является единственной непосредственной переменной л.-а. функции $f$ и $\mu_{1} \notin \tilde{M}_{i}^{(1)}$.

Если $\mu_{1}(0)=0$, то из функции

$$
g^{\prime}\left(f\left(x_{1}, \nu, \nu, \ldots, \nu\right), x_{2}\right),
$$


как и в случае 1 , с использованием операций композиции можно получить такую л.-а. функцию $\tilde{f}$, что $U(\tilde{f}) \notin M_{i}^{(1)}$, то есть выполнено $(28)$, что противоречит предположению.

Если $\mu_{1}(0) \neq 0$, то среди переменных $x_{2}, x_{3}, \ldots x_{n}$ л.-а. функции $f$ есть непосредственная переменная. Предположим, что $x_{2}-$ непосредственная переменная функции $f$. Тогда для л.-а. функции $f\left(x_{1}, x_{2}, \nu, \nu, \ldots, \nu\right)$ можно применить либо рассуждения случая 2.1 , если $\mu_{2} \notin \tilde{M}_{i}^{(1)}$, либо рассуждения случая 2.2 .1 , если $\mu_{2} \in \tilde{M}_{i}^{(1)}$.

Таким образом, $K(M)$ содержит $i$-сумматор $h\left(x_{1}, x_{2}\right)$,

$$
h=\nu_{1} x_{1}+\nu_{2} x_{2}+\nu_{0} .
$$

Пусть $f_{j} \in \mathfrak{L}_{k}$,

$$
f_{j}\left(x_{j, 1}, x_{j, 2}, \ldots, x_{j, n_{j}}\right)=\sum_{r=1}^{n_{j}} \mu_{j, r} x_{j, r}+\mu_{j, 0}
$$

$j=1,2$. Выберем произвольно $r_{j}, r_{j} \in\left\{1,2, \ldots, n_{j}\right\}, j=1,2$. Тогда, как нетрудно видеть,

$$
\begin{aligned}
\Psi_{i}\left(\mu_{1, r_{1}} \cdot \mu_{2, r_{2}}\right) \in \quad & \Psi_{i}\left(U \left(f _ { 1 } \left(x_{1,1}, x_{1,2}, \ldots, x_{1, r_{1}-1},\right.\right.\right. \\
& \left.\left.\left.f_{2}\left(x_{2,1}, x_{2,2}, \ldots, x_{2, n_{2}}\right), x_{1, r_{1}+1}, \ldots, x_{1, n_{1}}\right)\right)\right), \\
\Psi_{i}\left(\mu_{1, r_{1}}+\mu_{2, r_{2}}\right) \in \quad & \Psi_{i}\left(U \left(h \left(f_{1}\left(x_{1,1}, x_{1,2}, \ldots, x_{1, r_{1}}, \ldots, x_{1, n_{1}}\right)\right.\right.\right. \\
& \left.\left.\left.f_{2}\left(x_{2,1}, x_{2,2}, x_{2, r_{2}}, \ldots, x_{2, n_{2}}\right)\right)\right)\right),
\end{aligned}
$$

Отсюда и из соотношения (29) следует, что в $S(M)$ найдется такая л.-а. функция $f_{3}$,

$$
f_{3}=\sum_{j=1}^{n_{3}} \mu_{3, j} x_{3, j}+\mu_{3,0},
$$

что для некоторого $j_{0}, j_{0} \in\left\{1,2, \ldots, n_{3}\right\}$, и для некоторого $b, b \neq 0$,

$$
\Psi_{i}\left(\mu_{3, j_{0}}\right)=(0, b) .
$$

Положим

$$
f_{4}(x)=f_{3}(\underbrace{\nu, \nu, \ldots, \nu}_{j_{0}-1 \text { pa3 } \nu}, x, \nu, \nu, \ldots, \nu) .
$$

Тогда $U\left(f_{4}\right)=\left\{\mu_{3, j_{0}}\right\}$.

Пусть $T \in \mathbb{N}$. Применив операцию обратной связи к переменной $x_{2}$ л.-а. функции $h\left(x_{1}, f_{4}^{T}\left(x_{2}\right)\right)$, получим л.-а. функцию $f_{5, T}\left(x_{1}\right)$, для которой $U\left(f_{5, T}\right)=$ $\left\{\right.$ Об $\left.\left(\nu_{1}, \mu_{3, j_{0}}^{T}\right)\right\}$.

Найдется такое $T_{0}$, что Об $\left(\nu_{1}, \mu_{3, j_{0}}^{T_{0}}\right) \nsubseteq M_{i}^{(1)}$. Получили противоречие с предположением, что (28) не выполнено. Лемма доказана.

Теорема 6. Проблема проверки $K$-полноты конечных множеств из $\mathfrak{L}_{k}$ алгоритмически разрешима. 
Доказательство. Рассмотрим конечное множество $M$ л.-а. функций. Сначала проверим соотношение (3) для каждого $\Theta, \Theta \in J_{k}^{\prime}$. Если хотя бы одно из этих соотношений не выполнено, то по теореме 1 множество $M$ не является $K$-полным.

В противном случае проверяем соотношение (23). Для этого выбираем какое-либо $\eta, \eta \in U(M) \backslash E_{k}$ (если такого $\eta$ не существует, то $M$ не является $K$-полным) и проверяем равенство (11). Если оно не выполнено, то, согласно следствию из леммы 3 , включению $U(K(M)) \subseteq E_{p}(M)$ и теореме 4 , множество $M$ не является $K$-полным. Если равенство (11) имеет место, то проверяем справедливость включений $M \subseteq P_{s}^{(1)}$, $s=1,2, \ldots, l$. Выполнение хотя бы одного из них по теореме 4 означает, что $M$ не является $K$-полным.

Если ни одно из рассматриваемых включений не выполнено, то по теореме 4 остается проверить свойство (25).

Нетрудно видеть, что включения $U(K(M)) \subseteq M_{1}^{(1)}$ и $U(M) \subseteq M_{1}^{(1)}$ равносильны, причем второе из них несложно проверить.

Далее следующим образом убеждаемся в справедливости соотношений

$$
U(K(M)) \nsubseteq M_{i}^{(1)}
$$

для любого $i, i \in\{0,2,3, \ldots\}$. Для данного $i$ соотношение (30) следует из того, что $U(M) \nsubseteq M_{i}^{(1)}$.

Положим

$$
I=\left\{i \mid U(M) \subseteq M_{i}^{(1)}\right\}
$$

При $i \in I$ по лемме 11 соотношение (30) выполнено, если имеют место (29) и (27). Рассмотрим л.-а. функцию $f, f \in M \backslash V_{1}$. Пусть справедливо разложение (1) и, без ограничения общности, $x_{1}, x_{2}$ - непосредственные переменные функции $f$.

Пусть $\mu_{1}=\frac{u_{1}}{v_{1}}$. Если неприводимый многочлен $p_{j}, j \in\{2,3, \ldots\}$, не делит $u_{1}$, то $f \notin R_{j}^{\mathrm{c}}$ и $f \notin R_{j}^{\mathrm{H}}$. Поэтому можно проверить, верно ли свойство:

$$
\forall i, i \in\{0,2,3, \ldots\},\left(M \nsubseteq R_{i}^{\mathrm{c}} \wedge M \nsubseteq R_{i}^{\mathrm{H}}\right) .
$$

Если это свойство не выполнено, то по теореме 4 множество $M$ не является $K$-полным.

В противном случае множество

$$
\left\{j \mid \eta-\eta^{k} \notin W_{j}^{(1)}\right\}
$$

конечно. Поэтому свойство $(\forall j \in I$ выполнено (29)) может быть проверено. Теорема доказана.

\section{Список литературы}

1. Ван дер Варден Б. Л., Алгебра, пер. с нем., Наука, Москва, 1976, 648 с.

2. Гилл А., Линейнъе последовательностные машины, пер. с англ., Наука, Москва, 1974, $288 \mathrm{c.}$

3. Зарисский О., Самюэль П., Коммутативная алгебра, пер. с англ., ИЛ, Москва, 1963, 373 с.

4. Кудрявцев В. Б., Алешин С. В., Подколзин А. С., Введение в теорию автоматов, Наука, Москва, 1985, 320 с. 
5. Ленг С., Алгебра, пер. с англ., Мир, Москва, 1968, 564 с.

6. Лидл Р., Нидеррайтер Г., Конечные поля., пер. с англ., 1, Мир, Москва, 1988, 430 с.

7. Часовских А. А., "Условия полноты линейно-р-автоматных функций", Интеллектуальные системы, 18:3 (2014), 203-252.

8. Lau D., Function Algebras on Finite Sets. A Basic Course on Many-Valued Logic and Clone Theory, Springer, Rostok, 2006, 668 c.

9. Szendrei Á., "On closed classes of quasilinear functions", Chechoslovak Math. J., 30:3 (1980), 498-509.

Статья поступила 17.03.2015. 